\title{
Preoperative Plasma Fibrinogen Level: An Independent Predictor for Survival in Adult Patients With Xp11.2 Translocation Renal Cell Carcinoma
}

Jie Dong

Peking Union Medical College Hospital

Weifeng Xu

Peking Union Medical College Hospital

Zhigang Ji ( $\sim$ pumchjizhigang@163.com )

Peking Union Medical College Hospital https://orcid.org/0000-0001-9741-539X

Boju Pan

Peking Union Medical College Hospital

Research article

Keywords: Xp11.2 translocation, renal cell carcinoma, fibrinogen, prognosis, predictor

Posted Date: October 9th, 2020

DOl: https://doi.org/10.21203/rs.3.rs-88927/v1

License: (c) (i) This work is licensed under a Creative Commons Attribution 4.0 International License.

Read Full License 


\section{Abstract}

\section{Background}

Xp11.2 translocation renal cell carcinoma (Xp11.2 RCC) is a rare malignancy which is more common in children than in adults. It manifests with an aggressive course in adults and relatively indolent in children. Prognostic studies for adult patients are scare for the rarity of the disease; and the prognostic value of preoperative plasma fibrinogen level awaits further illumination.

\section{Methods}

This retrospective single-center study enrolled 24 consecutive newly diagnosed Xp11.2 RCC adult patients. Clinical presentations, baseline laboratory results and follow-up data were collected. Possible risk factors for progression free survival (PFS) and overall survival (OS) were first scanned with chisquare tests and t-tests to compare patients who suffered from progression or death and who did not. Independent risk factors for survival were further investigated with multivariate Cox regression.

\section{Results}

Twenty-four adult patients (median age 32, range 16-73), with a male-to-female ratio of $12: 12$, was included from 2010.4 to 2020.3. After a mean follow-up of 35.7 months, seven patients died. With univariate analysis, higher $\mathrm{C}$-reactive protein-to-albumin ratio $(p=0.028)$, higher baseline fibrinogen level $(p=0.006)$, and presence of distant metastasis $(p=0.007)$ were associated with progression of disease; higher preoperative fibrinogen level $(p=0.014)$ and distant metastasis $(p=0.020)$ were associated with death. With multivariate Cox regression, only baseline fibrinogen level $(p=0.001)$ was identified as an independent risk factor for progression free survival; meanwhile, fibrinogen level $(p=0.048)$ and distant metastasis $(p=0.043)$ were identified as independent risk factors for survival.

\section{Conclusions}

Preoperative plasma fibrinogen level, a routinely tested parameter before surgery, is a promising tool for risk stratification in adult patients with Xp11.2 RCC.

\section{Introduction}

Xp11.2 translocation renal cell carcinoma (XP11.2 RCC) is a rare and unique subtype of renal cell carcinoma characterized by translocations involving TFE3 gene ${ }^{[1-3]}$. It has been classified as a distinct clinical entity in the World Health Organization renal tumor classification (2004) and is now regarded as an important subtype of RCC, especially in children ${ }^{[4,5]}$. Compared to other subtypes of RCC, this malignancy manifests with more aggressive clinicopathologic features at diagnosis ${ }^{[5]}$ and worse prognosis ${ }^{[3,6]}$. In regard to the risk of disease re-occurrence and death, it is important to carry out prognostic studies to identify potential preoperative risk factors for disease progression and death which 
might help to guide interventions in the future. Moreover, prognostic researches for adult patients, should be especially encouraged due to at least three reasons: firstly, as a rare disease which mainly affects children ${ }^{[4]}$, data for adult patients is scarce for the time being which awaits further illumination; secondly, this disease has a more aggressive behavior in adult patients than in children ${ }^{[3]}$ which suggests a possibly different prognostic features in adults; thirdly, prior prognostic studies, which revealed several possible risk factors as neutrophil-to-lymphocyte ratio(NLR), C-reactive protein/albumin ratio(CRP/Alb ratio), platelet-to-lymphocyte ratio(PLR), tumor stage as well as inferior vena cava tumor thrombosis, included both child and adult patients ${ }^{[6,7]}$ for analysis which might bring bias. Therefore, it would be of importance to identify novel prognostic factors in adult XP11.2 RCC patients.

Fibrinogen, a routinely tested parameter included in the preoperative coagulation examination, is a glycoprotein synthesized by hepatocytes which participates in the process of blood coagulation. It also plays an important role in both inflammatory responses and tumor metastasis ${ }^{[8,9]}$. Elevated fibrinogen levels have been repeatedly reported to be associated with distant cancer metastasis and worse prognoses in many malignancies ${ }^{[8,10]}$, including RCC ${ }^{[11,12]}$. However, the potential role of preoperative plasma fibrinogen level for prognosis in XP11.2 RCC patients has not been investigated. Herein, we embarked on a study to examine the prognostic potential of preoperative fibrinogen level for newly diagnosed XP11.2 RCC.

\section{Patients And Methods}

\section{Patients}

Between April 2010 and March 2020, patients diagnosed with XP11.2 RCC after percutaneous renal mass biopsy or nephrectomy (radical or partial) at XXXX were included. Clinical information was retrieved from medical records. Pathological reports were carefully reviewed and TFE3 immunohistochemistry staining results were reconfirmed. The inclusion criteria were: 1 ) typical morphological features plus moderate-tostrong nuclear positivity with immunohistochemistry staining results for TFE3; 2) complete blood laboratory tests including blood routine test, blood biochemistry tests and coagulation test within one week before biopsy or nephrectomy; 3 ) adult patients defined as 16 years or older. The exclusion criteria were: pregnant women; $<16$ years old; with other inflammatory disease or a second tumor. This study was approved by the Ethics Committee of our hospital which was carried out in accordance with relevant guidelines and regulations. Informed consent was achieved from participants.

Demographic, clinical, laboratory, along with treatment-related data, including age at the time of diagnosis, gender, symptoms at presentation, serological findings, radiological results, and treatment options were documented. Preoperative neutrophil count, lymphocyte count, platelet count, hemoglobin level, C-reactive protein(CRP) level, albumin (Alb) level, lactic dehydrogenase (LDH) level, fibrinogen (Fbg) level were collected. Possible prognostic factors as NLR(neutrophil-to-lymphocyte ratio), PLR(platelet-tolymphocyte ratio), CRP/Alb ratio ${ }^{[6]}$ were calculated. Presence of tumor thrombus of IVC (inferior vena cava), lymph node metastasis and distant metastasis were confirmed from radiological examinations. 


\section{Follow-up}

Patients were followed until March, 2020 and follow-up data were analyzed by two independent urologists to determine progression. Progression was defined as tumor relapse, enlargement of tumor mass or presence of new metastatic lesions. Progression-free survival (PFS) was defined as the time from diagnosis to progression as previously defined or death from any cause. Overall survival (OS) was calculated as the time from diagnosis of XP11.2 RCC to death from any cause.

\section{Statistical Analysis}

With the help of SPSS 13.0 (SPSS Inc., Chicago, IL) which was used for statistical analysis, possible risk factors for PFS and OS were firstly scanned with chi-square tests (categorical covariates) and t-tests (continuous covariates) to compare patients who suffered from progression (or death) and who did not. NLR, PLR and CRP/Alb were transcoded into categorical variables with cutoff values set at 2.45, 140 and 0.083 respectively according to a prior study ${ }^{[6]}$. Variables with $p<0.10$ revealed by univariate comparison would further enter subsequent multivariate Cox regression. A stepwise regression method with a backward strategy (criteria for entry and removal of variables were $p<0.05$ and $p>0.10$ ) was utilized with one variable eliminated at a time. Parameters with $\mathrm{p}<0.05$ after multivariate scanning were considered as independent predictors of PFS or OS. Dichotomous variables which were showed to be independent risk factors were further analyzed with Kaplan-Meier method for depicting survival curves using log rank tests for comparison. For the convenience of survival curve depicting, continuous variables revealed as independent risk factors via multivariate Cox regression were transcoded into dichotomous variables with cutoff values set according to ROC curve analysis.

\section{Results}

\section{Patient Characteristics}

In total, 4958 cases of adult RCC were diagnosed during the past ten years in our center. Of them, 24 patients were diagnosed as XP 11.2 RCC with an overall proportion of $0.48 \%$. Table 1 outlined clinical characteristics of these 24 patients, including 12 (50.0\%) males and 12 (50.0\%) females with a male-tofemale ratio of 1:1. The median age at diagnosis of XP 11.2 RCC was 32 years (range, 16-73 years). Eleven patients (45.8\%) were symptomatic at the time of diagnosis while 13 patients (54.2\%) were asymptomatic. Tumors were found on 15 right kidneys (62.5\%) and 9 left kidneys (37.5\%) with a mean tumor size (maximum diameter) of $8.05 \pm 5.13 \mathrm{~cm}$. A large proportion of patients showed evidence of advanced stage at diagnosis: $45.8 \%$ patients with lymph node metastasis, 25\% patients with distant metastasis, $20.8 \%$ patients with tumor thrombus of IVC. Two patients did not receive further surgery after percutaneous renal mass biopsy-proven diagnosis. Twenty-two patients underwent nephrectomy (radical $16 / 22$ or partial 6/22). With a mean follow-up of 35.7 months, nine patients had disease progression and seven patients died. The estimated 3 year PFS was $66 \%$ and 3 year OS was $88.1 \%$. 
With univariate analysis (Table 2$)$, higher CRP/Alb ratio $(p=0.028)$, higher baseline fibrinogen $(p=0.006)$, and presence of distant metastasis $(p=0.007)$ were showed to be associated with progression of disease. All of them, with a $p$ value less than 0.10 , were incorporated into the multivariate Cox regression with a backward stepwise method. At the last step (Table 2), baseline fibrinogen level (HR 5.761; $95 \%$ confidence interval(Cl) $1.958-16.949 ; \mathrm{p}=0.001$ ) was identified as the only independent risk factor for PFS.

\section{Risk factors associated with overall survival}

Univariate analysis revealed four candidates $(p<0.10)$ for further multivariate regression: CRP/Alb $(p=0.053)$, preoperative fibrinogen $(p=0.014)$, tumor thrombus of IVC $(p=0.088)$ and distant tumor metastasis $(p=0.020)$. Of them, fibrinogen level and presence of distant metastasis were showed as statistically significant risk factors $(\mathrm{p}<0.05)$ for death using univariate scanning. After multivariate analysis, preoperative fibrinogen level (HR 2.954; 95\% Cl 1.011-8.629; $p=0.048)$ and distant tumor metastasis (HR 12.287; 95\% Cl 1.083-139.409; $p=0.043$ ) were identified as independent prognostic factors for survival.

\section{Survival curves}

According to ROC curve analysis, the AUC (area under the curve) value of preoperative fibrinogen level of overall survival was $0.861(p=0.006)$. The optimal cut-off value for preoperative fibrinogen was $3.84 \mathrm{~g} / \mathrm{L}$. Fibrinogen (Fbg), a continuous variable, was then transcoded into a dichotomous variable ( $\mathrm{Fbg} \geq 3.84 \mathrm{~g} / \mathrm{L}$ versus $\mathrm{Fbg}<3.84 \mathrm{~g} / \mathrm{L}$ ) for survival curve depicting. Fig 1 a showed a statistical significant difference of PFS $(p=0.0006)$ between patients with and without elevated $(\geq 3.84 \mathrm{~g} / \mathrm{L})$ fibrinogen: the median PFS was not reached for the $\mathrm{Fbg}<3.84 \mathrm{~g} / \mathrm{L}$ group compared to 34 months in patients with elevated fibrinogen. Fig $1 \mathrm{~b}$ revealed a significant difference of $\mathrm{OS}(\mathrm{p}=0.0417)$ between these two groups: median survival 56 months $(\mathrm{Fbg}<3.84 \mathrm{~g} / \mathrm{L})$ versus. 48 months $(\mathrm{Fbg} \geq 3.84 \mathrm{~g} / \mathrm{L})$.

Presence of distant metastasis, a dichotomous variable which was shown to be both a risk factor for progression with univariate analysis and an independent risk factor for OS with multivariate analysis, was further used to delineate survival curves (Fig 2). Significant difference was noted for PFS ( $p=$ 0.0082): median survival for patients without distant metastasis was 46 months compared with 22 months in patients with distant metastasis (Fig 2a). Fig 2b showed the survival curves for OS for patients with and without distant metastasis (median survival 48 months versus. 56 months, $p=0.025$ ).

\section{Discussion}

XP11.2 RCC accounts for $20-40 \%$ of pediatric RCC ${ }^{[13]}$ and only $0.72-1.6 \%$ of adult RCC ${ }^{[5,14]}$. This subtype of RCC in adults requires special attention and more intensive researches for its rarity, aggressiveness in nature ${ }^{[3,13]}$ and possible different treatment options (eg. m-TOR inhibitors or VEGFtargeted agents) ${ }^{[16,17]}$. This single-center, retrospective study identified an overall incidence of $0.48 \%$ for XP11.2 translocation RCC out of all adult RCCs based on a ten-year data. This result was consistent with another Asian cohort ${ }^{[5]}$ ( $0.72 \%$ in Korea) which further demonstrated the rarity of this disease in adults. 
Although a prior meta-analysis suggested a female gender predominance in adult XP11.2 translocation RCC ${ }^{[13]}$, possibly due to its X-chromosome related nature, our study found an equal gender distribution as observed in children which might be explained by the absence of translocation on the Barr body (inactive $\mathrm{X}$ chromosome) or by the relatively limited number of patients enrolled. Moreover, consistent with previous reports ${ }^{[7,18]}$ and for unknown reasons, right side prevalence was observed in our cohort.

Several attempts have been made to investigate the possible risk factors for survival ${ }^{[6,7]}$ which suggest several possible risk factors as NLR, CRP/Alb, PLR and tumor thrombosis of IVC and tumor stage. However, disparities are noticed in different studies and these factors have never been externally validated. Moreover, these studies enrolled both children and adult patients which might hinder the accuracy of the prediction model as children present with relatively indolent disease course ${ }^{[3]}$. This study, according to our limited knowledge, is one of the first endeavors to validate those previously reported prognostic factors and to explore novel potential risk factors in adults with XP11.2 RCC. According to this study, previously reported risk factors as CRP/Alb ${ }^{[6]}$, tumor thrombosis of IVC ${ }^{[7]}$ showed statistical significance or borderline significance (Table 2 and Table 3) with univariate analysis; and distant metastasis, a parameter reflecting tumor stage, was showed to be an independent risk factor for OS with multivariate analysis. Moreover, preoperative plasma fibrinogen level, a parameter routinely examined preoperatively but never tested for risk stratification in XP11.2 RCC, was demonstrated as an independent risk factor for both PFS and OS.

Elevated fibrinogen levels have been linked to poor outcomes in many types of malignancies, including kidney cancers ${ }^{[8-9,11-12]}$. However, its role in predicting survival in XP11.2 RCC has not been well illuminated. In our retrospective study, we not only demonstrated its independent nature to predict progression, but also suggested a crucial role to predict overall survival. There have been several theories to explain the association between fibrinogen level and outcomes of malignancies: firstly, high fibrinogen level might be a reflection of tumor induced systemic inflammatory response ${ }^{[19]}$; secondly, fibrinogen can

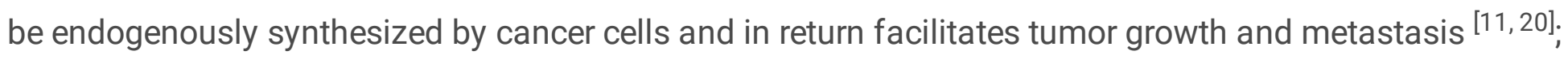
thirdly, fibrinogen could activate cancer cell adhesion with platelets to form a dense fibrin 'protective' layer outside tumor cells from natural killer cells ${ }^{[21]}$. Aside from these common pathways, there might be another two distinct mechanisms to clarify the association between fibrinogen and outcomes of XP11.2

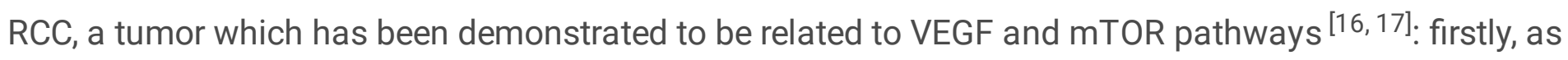
an extracellular matrix element, fibrinogen can regulate growth of tumor cells by binding to VEGF $^{[22]}$; secondly, fibrinogen might promote cell motility by inducing epithelial-mesenchymal transition via the pAKT / p-mTOR pathway ${ }^{[23]}$. These mechanisms suggest a possible internal link between fibrinogen and this unique subtype of RCC and might explain the strong association found with a relatively small sample size.

Our study has limitations: first of all, TFE3 break-apart FISH analysis was not done for this cohort of patients. However, as TFE3 immunohistochemistry was also an accurate tool for diagnosis which had been accepted for prior studies ${ }^{[3,6]}$, we still utilized TFE3 immunohistochemistry stain as our inclusion 
criteria; second, the results of this study were not externally validated, future work focusing on the role of fibrinogen in XP11.2 translocation RCC might be helpful.

In conclusion, preoperative plasma fibrinogen level, a routinely tested parameter before surgery, might be a potential tool for risk stratification in adults with Xp11.2 RCC.

\section{Abbreviations}

RCC: Renal cell carcinoma

Fbg: Fibrinogen

NLR: Neutrophil-to-lymphocyte ratio

PLR: Platelet-to-lymphocyte ratio

PFS: Progression-free survival

OS: Overall survival

\section{Declarations}

Ethics approval $\mathrm{T}$ This study was approved by the Ethics Committee of PUMCH.

Patient consent for publication Informed consent for publication was achieved from participants.

Availability of data and materials $\square$ Data and materials generated during and/or analysed during this study are available from the corresponding author (Ji Zhigang) on reasonable request.

Authors' contributions $\square$ JD, WFX, ZGJ performed the research and collected clinical data; BJP reviewed the pathologies; JD wrote the manuscript; WFX and ZGJ supervised the study and revised the manuscript.

Competing interests $\square$ The authors of this manuscript declare no conflict of interest.

Funding] Fundamental Research Funds for the Central Universities (3332020004, for DJ)

Acknowledgements $\square$ Not applicable.

\section{References}

[1] Argani P, Ladanyi M. Translocation carcinomas of the kidney. Clin Lab Med. 2005 Jun;25(2):363-378.

[2] Ellis CL, Eble JN, Subhawong AP, et al. Clinical heterogeneity of Xp11

translocation renal cell carcinoma: impact of fusion subtype, age, and stage. Mod Pathol. 2014 Jun;27(6):875-886. 
[3] Kuthi L, Somorácz Á, Micsik T, et al. Clinicopathological Findings on 28 Cases with XP11.2 Renal Cell Carcinoma. Pathol Oncol Res. 2020 Jan 18. [Epub ahead of print]. doi: 10.1007/s12253-019-00792-0.

[4] Argani P, Lal P, Hutchinson B, Lui MY, Reuter VE, Ladanyi M. Aberrant nuclear immunoreactivity for TFE3 in neoplasms with TFE3 gene fusions: a sensitive and specific immunohistochemical assay. Am J Surg Pathol 2003; 27:750-61.

[5] Choo MS, Jeong CW, Song C, et al. Clinicopathologic Characteristics and Prognosis of Xp11.2 Translocation Renal Cell Carcinoma: Multicenter, Propensity Score Matching Analysis. Clin Genitourin Cancer. 2017 Oct;15(5):e819-e825.

[6] Agizamhan S, Qu F, Liu N, et al. Preoperative neutrophil-to-lymphocyte ratio predicts the surgical outcome of Xp11.2 translocation/TFE3 renal cell carcinoma patients. BMC Urol. 2018 Jun 11;18(1):60.

[7] Liu N, Wang Z, Gan W,et al. Renal Cell Carcinoma Associated with Xp11.2 Translocation/TFE3 Gene Fusions: Clinical Features, Treatments and Prognosis. PLoS One. 2016 Nov 28;11(11):e0166897.

[8] Takeuchi H, Ikeuchi S, Kitagawa Y et al. Pretreatmentplasma fibrinogen level correlates with tumor progression and metastasis in patients with squamous cell carcinoma of the esophagus. J Gastroenterol Hepatol 2007; 22: 2222-2227.

[9] Palumbo JS, Kombrinck KW, Drew AF, et al. Fibrinogen is an important determinant of the metastatic potential of circulating tumor cells. Blood. 2000;96: 3302-3309.

[10] Fan S, Guan Y, Zhao G, et al. Association between plasma fibrinogen and survival in patients with small-cell lung carcinoma. Thorac Cancer. 2018 Jan;9(1):146-151.

[11] Tian Y, Hong M, Jing S, et al. Clinical and Prognostic Effect of Plasma Fibrinogen in Renal Cell Carcinoma: A Meta-Analysis. Biomed Res Int. 2017;2017:9591506.

[12] He X, Huang T, Xue Y, et al. Association of Preperative Plasma D-dimmer and Fibrinogen and Renal Cell CarcinomaOutcome. J Cancer. 2019 Jul 10;10(17):4096-4105.

[13] Cheng X, Gan W, Zhang G, et al. Clinical characteristics of XP11.2 translocation/TFE3 gene fusion renal cell carcinoma: a systematic review and meta-analysis of observational studies. BMC Urol. 2016 Jul 11;16(1):40.

[14] Komai Y, Fujiwara M, Fujii Y, et al. Adult Xp11 translocation renal cell carcinoma diagnosed by cytogenetics and immunohistochemistry. Clin Cancer Res. 2009 Feb 15;15(4):1170-1176.

[15] Qu Y, Gu C, Wang H, et al. Diagnosis of adults Xp11.2 translocation renal cell carcinoma by immunohistochemistry and FISH assays: clinicopathological data from ethnic Chinese population. Sci Rep. 2016 Feb 16;6:21677. 
[16] Malouf GG, Camparo P, Oudard S, et al. Targeted agents in metastatic Xp11 translocation/TFE3 gene fusion renal cell carcinoma (RCC): a report from the Juvenile RCC Network. Ann Oncol. 2010 Sep;21(9):1834-1838.

[17] Choueiri TK, Lim ZD, Hirsch MS, et al. Vascular endothelial growth factor-targeted therapy for the treatment of adult metastatic Xp11.2 translocation renal cell carcinoma. Cancer. $2010 \mathrm{Nov}$ 15;116(22):5219-5225.

[18] Hirobe M, Masumori N, Tanaka T, et al. Clinicopathological characteristics of Xp11.2 translocation renal cell carcinoma in adolescents and adults: Diagnosis using immunostaining of transcription factor E3 and fluorescence in situ hybridization analysis. Int J Urol. 2016 Feb;23(2):140-145.

[19] Lip GY, Chin BS, Blann AD. Cancer and the prothrombotic state. Lancet Oncol. 2002 Jan;3(1):27-34.

[20]Simpson-Haidaris PJ, Rybarczyk B. Tumors and fibrinogen. The role of fibrinogen as an extracellular matrix protein. Ann N Y Acad Sci. 2001;936:406-425.

[21] Zheng S, Shen J, Jiao Y, et al. Platelets and fibrinogen facilitate each other in protecting tumor cells from natural killercytotoxicity. Cancer Sci. 2009 May;100(5):859-865.

[22] Sahni A, Francis CW. Vascular endothelial growth factor binds to fibrinogen and fibrin and stimulates endothelial cellproliferation. Blood. 2000 Dec 1;96(12):3772-3778.

[23] Zhang F, Wang $Y$, Sun $P$, et al. Fibrinogen promotes malignant biological tumor behavior involving epithelial-mesenchymal transition via the p-AKT/p-mTOR pathway in esophageal squamous cell carcinoma. J Cancer Res Clin Oncol. 2017 Dec;143(12):2413-2424.

\section{Tables}


Table 1. Clinical characteristics of patients with Xp11.2 translocation RCC

Value $\%$

Gender

\begin{tabular}{|c|c|c|c|}
\hline & Male & 12 & $50 \%$ \\
\hline & Female & 12 & $50 \%$ \\
\hline Age (year) & & $42.7 \pm 14.9$ & \\
\hline \multicolumn{4}{|c|}{ Symptoms at onset } \\
\hline & Symptomatic & 11 & $45.80 \%$ \\
\hline & Asymptomatic & 13 & $54.20 \%$ \\
\hline \multicolumn{4}{|l|}{ Location } \\
\hline & Right & 15 & $62.50 \%$ \\
\hline & Left & 9 & $37.50 \%$ \\
\hline Tumor size $(\mathrm{cm})$ & & $8.05 \pm 5.13$ & \\
\hline \multicolumn{4}{|c|}{ Lymph node metastasis } \\
\hline & Positive & 11 & $45.80 \%$ \\
\hline & Negative & 13 & $54.20 \%$ \\
\hline \multicolumn{4}{|c|}{ Distant metastasis } \\
\hline & Positive & 6 & $25.00 \%$ \\
\hline & Negative & 18 & $75.00 \%$ \\
\hline \multicolumn{4}{|c|}{ Tumor thrombus of IVC } \\
\hline & Positive & 5 & $20.80 \%$ \\
\hline & Negative & 19 & $79.20 \%$ \\
\hline \multicolumn{4}{|c|}{ Surgical treatment } \\
\hline & No surgery & 2 & $8.30 \%$ \\
\hline & Partical & 6 & $25.00 \%$ \\
\hline & Radical & 16 & $66.70 \%$ \\
\hline
\end{tabular}


Table 2. Risk factors for disease progression in patients with Xp11.2 translocation RCC

\begin{tabular}{|c|c|c|c|c|c|}
\hline & \multicolumn{3}{|c|}{ Univariate analysis } & \multicolumn{2}{|c|}{$\begin{array}{l}\text { Multivariate Cox regression (last } \\
\text { step) }\end{array}$} \\
\hline & Progression & $\begin{array}{l}\text { No } \\
\text { progession }\end{array}$ & $\mathrm{P}$ & $\mathrm{HR}(95 \% \mathrm{Cl})$ & $\mathrm{P}$ \\
\hline Gender,male (n) & $4 / 9$ & $8 / 15$ & 0.673 & & \\
\hline Age (year) & $46.6 \pm 17.7$ & $40.4 \pm 13.2$ & 0.340 & & \\
\hline $\begin{array}{l}\text { Symptomatic patient } \\
\text { (n) }\end{array}$ & $6 / 9$ & $5 / 15$ & 0.113 & & \\
\hline Right side (n) & $5 / 9$ & $4 / 15$ & 0.157 & & \\
\hline Tumor size $(\mathrm{cm})$ & $9.13 \pm 5.82$ & $7.40 \pm 4.76$ & 0.435 & & \\
\hline$N L R>2.45$ & $7 / 9$ & $8 / 15$ & 0.231 & & \\
\hline PLR>140 & $4 / 9$ & $9 / 15$ & 0.459 & & \\
\hline CRP/Alb $>0.083$ & $5 / 9$ & $2 / 15$ & 0.028 & & \\
\hline LDH (U/L) & $255.3 \pm 101.8$ & $201.0 \pm 104.8$ & 0.227 & & \\
\hline Hemoglobin $(\mathrm{g} / \mathrm{L})$ & $123.2 \pm 23.3$ & $132.8 \pm 20.7$ & 0.306 & & \\
\hline $\mathrm{Fbg}(\mathrm{g} / \mathrm{L})$ & $3.95 \pm 1.04$ & $2.69 \pm 0.47$ & 0.006 & $5.761(1.958-16.949)$ & 0.001 \\
\hline $\begin{array}{l}\text { Tumor thrombus of } \\
\text { IVC }\end{array}$ & $3 / 9$ & $2 / 15$ & 0.243 & & \\
\hline $\begin{array}{l}\text { Lymph node } \\
\text { metastasis }\end{array}$ & $6 / 9$ & $5 / 15$ & 0.113 & & \\
\hline Distant metastasis & $5 / 9$ & $1 / 15$ & 0.007 & & \\
\hline Radical surgery * & $5 / 7$ & $11 / 15$ & 0.926 & & \\
\hline
\end{tabular}


Table 3. Risk factors for death in patients with Xp11.2 translocation RCC

\begin{tabular}{|c|c|c|c|c|c|}
\hline & \multicolumn{3}{|c|}{ Univariate analysis } & \multicolumn{2}{|c|}{$\begin{array}{l}\text { Multivariate Cox regression (last } \\
\text { step) }\end{array}$} \\
\hline & Death & No death & $\mathrm{P}$ & $\operatorname{HR}(95 \% \mathrm{Cl})$ & $P$ \\
\hline Gender,male (n) & $4 / 7$ & $8 / 17$ & 0.653 & & \\
\hline Age (year) & $46.4 \pm 19.7$ & $41.2 \pm 12.9$ & 0.446 & & \\
\hline $\begin{array}{l}\text { Symptomatic patient } \\
\text { (n) }\end{array}$ & $4 / 7$ & $7 / 17$ & 0.476 & & \\
\hline Right side (n) & $4 / 7$ & $5 / 17$ & 0.202 & & \\
\hline Tumor size $(\mathrm{cm})$ & $8.87 \pm 6.47$ & $7.71 \pm 4.66$ & 0.626 & & \\
\hline$N L R>2.45$ & $5 / 7$ & $10 / 17$ & 0.562 & & \\
\hline PLR $>140$ & $2 / 7$ & $11 / 17$ & 0.106 & & \\
\hline CRP/Alb $>0.083$ & $4 / 7$ & $3 / 17$ & 0.053 & & \\
\hline $\mathrm{LDH}(\mathrm{U} / \mathrm{L})$ & $239.9 \pm 107.8$ & $213.8 \pm 106.1$ & 0.591 & & \\
\hline Hemoglobin $(\mathrm{g} / \mathrm{L})$ & $122.1 \pm 23.2$ & $132.1 \pm 21.1$ & 0.317 & & \\
\hline $\mathrm{Fbg}(\mathrm{g} / \mathrm{L})$ & $4.16 \pm 1.10$ & $2.76 \pm 0.47$ & 0.014 & $2.954(1.011-8.629)$ & 0.048 \\
\hline $\begin{array}{l}\text { Tumor thrombus of } \\
\text { IVC }\end{array}$ & $3 / 7$ & $2 / 17$ & 0.088 & & \\
\hline $\begin{array}{l}\text { Lymph node } \\
\text { metastasis }\end{array}$ & $5 / 7$ & $6 / 17$ & 0.106 & & \\
\hline Distant metastasis & $4 / 7$ & $2 / 17$ & 0.020 & $12.287(1.083-139.409)$ & 0.043 \\
\hline Radical surgery * & $4 / 6$ & $12 / 16$ & 0.696 & & \\
\hline
\end{tabular}

\section{Figures}



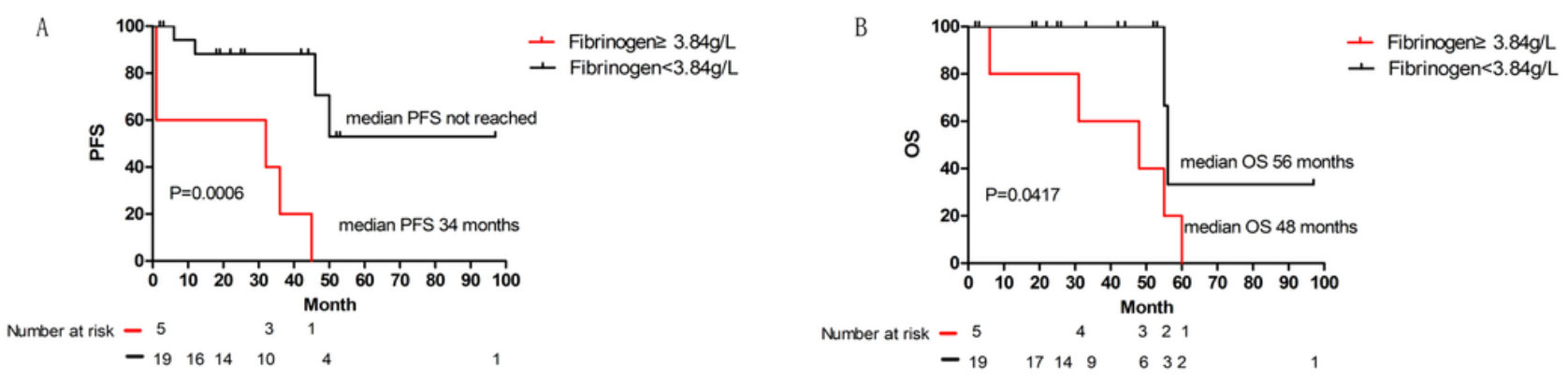

\section{Figure 1}

Survival curves of PFS (Fig 1a) and OS (Fig 1b) with a cutoff point of fibrinogen at 3.84g/L

A

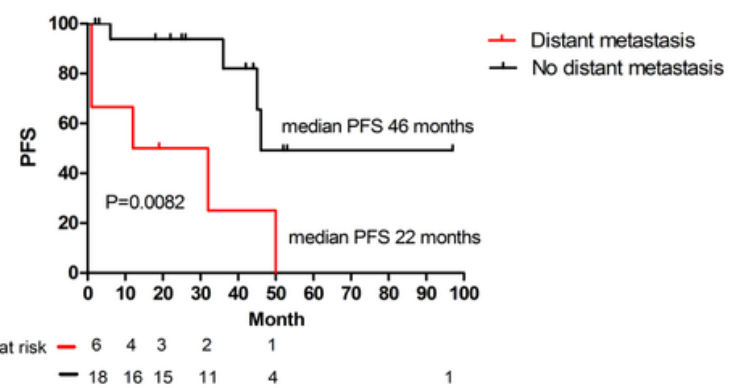

B

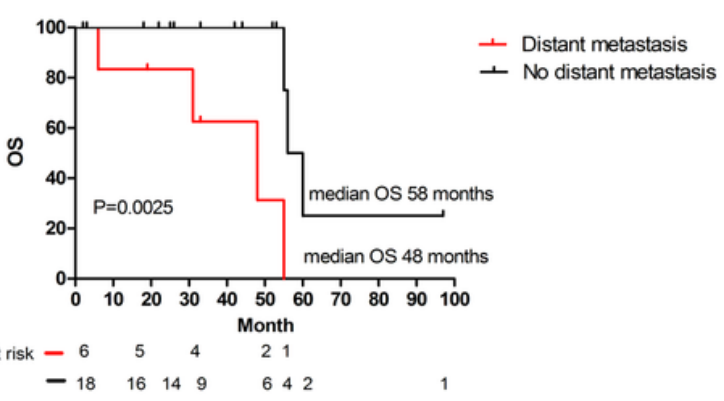

Figure 2

Survival curves of PFS (Fig 2a) and OS (Fig 2b) for patients with and without distant metastasis 